\title{
Distributed Information Filters for MAV Cooperative Localization
}

\author{
Andrea Cristofaro, Alessandro Renzaglia and Agostino Martinelli
}

\begin{abstract}
This paper introduces a new approach to the problem of simultaneously localizing a team of micro aerial vehicles (MAV) equipped with inertial sensors able to monitor their motion and with exteroceptive sensors. The method estimates a delayed state containing the trajectories of all the MAVs. The estimation is based on an Extended Information Filter whose implementation is distributed over the team members. The paper introduces two contributions. The former is a trick which allows exploiting the information contained in the inertial sensor data in a distributed manner. The latter is the use of a projection filter which allows exploiting the information contained in the geometrical constraints which arise as soon as the MAV orientations are characterized by unitary quaternions. The performance of the proposed strategy is evaluated with synthetic data. In particular, the benefit of the previous two contributions is pointed out.
\end{abstract}

\section{Introduction}

In recent years, flying robotics has received significant attention from the robotics community. The ability to fly allows easily avoiding obstacles and quickly having an excellent birds eye view. These navigation facilities make flying robots the ideal platform to solve many tasks like exploration, mapping, reconnaissance for search and rescue, environment monitoring, security surveillance, inspection etc. In the framework of flying robotics, micro aerial vehicles (MAV) have a further advantage. Due to the small size they can also be used in narrow out- and indoor environment and they represent only a limited risk for the environment and people living in it. One of the main prerequisite for the successful accomplishment of many tasks is a precise vehicle localization. Since micro aerial vehicles are equipped with low

A. Cristofaro, INRIA Rhône-Alpes, Grenoble, France, e-mail: andrea.cristofaro@inrialpes.fr A. Renzaglia, INRIA Rhône-Alpes, Grenoble, France, e-mail: alessandro.renzaglia@inrialpes.fr A. Martinelli, INRIA Rhône-Alpes, Grenoble, France, e-mail: agostino.martinelli@inrialpes.fr 
computational capabilities an efficient solution must be able to distribute the computation among all the agents in order to exploit the computational resources of the entire team. Distributing the computation has also another key advantage. It allows us to make the solution robust with respect to failures. On the other hand, distributing the computation must also account for the limited communication capabilities. The cooperative localization problem was formulated in [10] and it has been faced by many authors so far. Fox and collaborators [3] introduced a probabilistic approach based on Markov localization. Their approach has been validated through real experiments showing a drastic improvement in localization speed and accuracy when compared to conventional single robot localization. Other approaches take advantage of relative observations for multi-robot localization $[4,5,9,15,16,19]$. In [5] a method based on a combination of maximum likelihood estimation and numerical optimization was introduced. This method allows to reduce the error in the robot localization by using the information coming from relative observations among the robots in the team. In [16], a distributed multi robot localization strategy was introduced. This strategy is based on an Extended Kalman Filter to fuse proprioceptive and exteroceptive sensor data. In [12], the same approach was adapted in order to deal with any kind of relative observations among the robots. In [16], it was shown that the equations can be written in a decentralized form, allowing the decomposition into a number of smaller communicating filters. However, the distributed structure of the filter only regards the integration of the proprioceptive data (i.e. the so called prediction phase). As soon as an observation between two robots occurs, communication between each member of the team and a single processor (which could be embedded in a member of the team) is required. The same communication skill is required when even an exteroceptive measurements which only regards a single robot occurs (e.g. a GPS measurement). Furthermore, the computation required to integrate the information coming from this observation is entirely performed by a single processor with a computational complexity which scales quadratically with the number of robots. Obviously, the centralized structure of the solution in dealing with exteroceptive observations becomes a serious inconvenience when the communication and processing capabilities do not allow to integrate the information contained in the exteroceptive data in real time. In particular, this happens as soon as the number of robots is large, even if each robot performs very few exteroceptive observations. In [13] this problem was considered. However, the structure of the filter was maintained the same as in [16] (namely centralized in dealing with exteroceptive data). Each robot was supposed to be equipped with several sensors and the optimal sensing frequencies were analytically derived by maximizing the final localization accuracy. The limit of this approach is that as the number of robots increases, the sensing frequencies reduce. In other words, by performing the estimation process in a centralized fashion it is necessary to reduce the number of observations to be processed as the number of robots increases. Hence, distibuting the entire estimation process can provide a great improvement. Very recently a decentralized cooperative localization approach has been presented in [11]. 
The information filter is very appealing in this framework since the integration of the exteroceptive data is very simple and could be easily distributed. On the other hand, the equations which characterize the prediction step are much more complex and their distributed implementation seems to be forbidden. This is a serious inconvenience since the proprioceptive data run at a very high frequency.

Eustice et al. [2] and Caballero et al. [1] have recently shown that by using a delayed state also the prediction step has some nice properties. In particular, in [2] a solution to the SLAM problem by using an Extended Information Filter (EIF) to estimate a delayed state has been proposed. In [1] the tracking problem has been considered.

In this paper we consider the problem of cooperative localization in $3 D$ when the MAVs are equipped with inertial sensors and exteroceptive sensors (e.g. range sensors and GPS). We adopt a delayed state and we perform its estimation by using an Extended Information Filter. We introduce a simple trick which allows us to mathematically express the quantities measured by the IMU (Inertial Measurement Unit) as a function of the delayed state (i.e. the state to be estimated). In other words, by using this trick, the link between sensor-state for the IMU (which are typically proprioceptive sensors) has the same mathematical expression of the one which characterizes an exteroceptive observation. This allows us to use the equations of the integration of the exteroceptive data also to integrate the IMU data. In this way the equations of the EIF prediction step are never used and the overall estimation process can be easily distributed.

The second contribution of this paper is related to another important issue which arises when dealing with a $3 D$ environment. The orientation of a MAV which moves in $3 D$ is provided by 3 parameters. On the other hand, the MAV dynamics become very easy by adopting quaternions. However, this parameterization is redundant. This means that part of the information is frozen in a geometrical constraint. Without using this constraint part of the information is not exploited and the overall precision gets worse. To the best of our knowledge, this issue has never been considered in the framework of flying robotics. On the other hand, the problem of exploiting the information contained in geometrical constraints is not new in the mobile robotics literature. In particular, it has been considered in SLAM when using a relative map. To this regard a new filter, the projection filter, has been introduced [14]. In this paper we will adopt the same approach. In particular, we consider the geometrical constraint (expressing that the quaternion must be unitary) as an ideal observation.

The paper is structured as follows. In Section 2 it is given a detailed description of the dynamics, the measurement model and the estimation process with the EIF for a single MAV. Section 3 is dedicated to the extension of the previous results to multi robot systems; in particular a distributed EIF algorithm is presented, taking into account relative observations between the robots. In Section 4 we present some simulation results to illustrate the efficiency of the estimation algorithm. 


\section{The Case of One Single MAV}

For the sake of clarity, we begin our analysis by the description of the model for a single MAV. The extension of the presented dynamics and measurement model to multi robot systems is straightforward.

\subsection{The System}

We provide here a mathematical description of our system. We introduce a global frame, whose $z$-axis is the vertical one. Let us consider a MAV equipped with IMU proprioceptive sensors (an accelerometer and a gyroscope) as well as some suitable exteroceptive sensors (GPS, range sensors). In this paper we assume that the IMU data are unbiased. From a practical point of view, unbiased data can be obtained by continuously calibrating the IMU sensors (see for instance [6]). The configuration of the MAV is described by a vector $(r, v, \theta) \in \mathbf{R}^{9}$ where $r=\left(r_{x}, r_{y}, r_{z}\right) \in \mathbf{R}^{3}$ is the position, $v=\left(v_{x}, v_{y}, v_{z}\right) \in \mathbf{R}^{3}$ is the speed and $\theta=\left(\theta_{\mathrm{r}}, \theta_{\mathrm{p}}, \theta_{\mathrm{y}}\right) \in \mathbf{R}^{3}$ assignes the MAV orientation: $\theta_{\mathrm{r}}$ is the roll angle, $\theta_{\mathrm{p}}$ is the pitch angle and $\theta_{\mathrm{y}}$ is the yaw angle. We will adopt lower case letters to express a quantity in the global frame, while capital letters for the same quantity expressed in the local frame (i.e. the one attached to the MAV).The system description can be simplified adopting a quaternions framework. We recall that the quaternions space $\mathbf{H}$ is the noncommutative set of elements

$$
\mathbf{H}=\left\{q_{t}+q_{x} i+q_{y} j+q_{z} k: \quad q_{t}, q_{x}, q_{y}, q_{z} \in \mathbf{R}, \quad i^{2}=j^{2}=k^{2}=i j k=-1\right\} .
$$

For an arbitrary quaternion $q=q_{t}+q_{x} i+q_{y} j+q_{z} k$, we define the conjugate element $q^{*}=q_{t}-q_{x} i-q_{y} j-q_{z} k$ and the norm $\|q\|=\sqrt{q q^{*}}=\sqrt{q^{*} q}=\sqrt{q_{t}^{2}+q_{x}^{2}+q_{y}^{2}+q_{z}^{2}}$.

Let us denote by $a_{g}$ the gravity acceleration (i.e. $a_{g}=-(0,0, g)$ with $\left.g \simeq 9.81 \mathrm{~m} / \mathrm{s}^{2}\right)$ and by $A, \Omega$ the acceleration and the angular speed provided by the IMU; regarding the acceleration, the one perceived by the accelerometer $(A)$ is not simply the MAV acceleration $(\bar{A})$ : it also contains the gravity acceleration $\left(A_{g}\right)$. In particular, we have $A=\bar{A}-A_{g}$ since, when the camera does not accelerate (i.e. $\bar{A}=0$ ) the accelerometer perceives an acceleration which is the same of an object accelerated upward in the absence of gravity.

The continuous-time dynamics of the MAV is given by the following system of ordinary differential equations

$$
\begin{gathered}
\dot{r}=v \\
\dot{v}=q \cdot \bar{A} \cdot q^{*}=q \cdot A \cdot q^{*}+a_{g}
\end{gathered}
$$




$$
\dot{q}=\frac{1}{2} q \cdot \Omega
$$

where $r, v, \Omega, A$ are purely imaginary quaternions, while $q$ is a unitary quaternion. The following relations for roll, pitch and yaw angles $\theta_{\mathrm{r}}, \theta_{\mathrm{p}}, \theta_{\mathrm{y}}$ hold

$$
\begin{aligned}
& \theta_{\mathrm{r}}=\frac{q_{t} q_{x}+q_{y} q_{z}}{1-2\left(q_{x}^{2}+q_{y}^{2}\right)} \\
& \theta_{\mathrm{p}}=q_{t} q_{y}-q_{x} q_{z} \\
& \theta_{\mathrm{y}}=\frac{q_{t} q_{z}+q_{y} q_{x}}{1-2\left(q_{y}^{2}+q_{z}^{2}\right)} .
\end{aligned}
$$

During the exploration, the MAV performs measurements thanks to its exteroceptive sensors equipment; such measurements can be individual (i.e. GPS-based measurements) as well as relative to other MAVs poses or to the position of fixed landmarks. The general single MAV observation equation is given by

$$
z=h(r, v, q)
$$

where $h(\cdot, \cdot, \cdot)$ is a known function.

In the case the exteroceptive sensor is a GPS, the observation equation is very simple as it is linear

$$
z_{G P S}=r
$$

\subsection{Estimation with the EIF: the integration of the exteroceptive data}

Let us denote with $\Sigma$ and $\xi$ the information matrix and the information vector respectively; in addition let $R$ be the covariance matrix characterizing the measurement error for an exteroceptive sensors. The update equations at the time step $i$ are (see [20]):

$$
\begin{gathered}
\Sigma_{i}=\bar{\Sigma}_{i}+\Sigma_{o b s}, \quad \Sigma_{o b s}=H_{i}^{T} R^{-1} H_{i}, \\
\xi_{i}=\bar{\xi}_{i}+\xi_{o b s}, \quad \xi_{o b s}=H_{i}^{T} R^{-1}\left[z_{i}-h\left(\bar{\mu}_{i}\right)+H_{i} \bar{\mu}_{i}\right],
\end{gathered}
$$

where $\bar{\Sigma}_{i}, \bar{\xi}_{i}$ are the predicted information matrix and information vector, $\bar{\mu}_{i}=$ $\bar{\Sigma}_{i}^{-1} \bar{\xi}_{i}$ is the predicted mean value and $H_{i}$ is the Jacobian of the observation function $h$ evaluated at $\bar{\mu}_{i}$. The structure of such equation is very simple as the update consists only in summing the new information from the exteroceptive sensors to the predicted values.

The case of GPS observations is even easier to treat; since the function $h$ is linear 
we have $h\left(\bar{\mu}_{i}\right)=H_{i} \bar{\mu}_{i}=\bar{\mu}_{i}$ and hence the update equation for the information filter is

$$
\xi_{i}=\bar{\xi}_{i}+H_{i}^{T} R^{-1} z_{i}
$$

In particular the explicit computation of the mean value is not performed and this is a key advantage since the information matrix inversion requires in general a high computational burden.

\subsection{Estimation with the EIF: the integration of the proprioceptive data}

Denoting by $Q$ a noise term affecting the system dynamics, the prediction steps are given by

$$
\begin{gathered}
\bar{\Sigma}_{i}=\left[F_{i} \Sigma_{i-1}^{-1} F_{i}^{T}+Q\right]^{-1}, \\
\bar{\xi}_{i}=\bar{\Sigma}_{i} F_{i} \Sigma_{i-1}^{-1} \xi_{i-1},
\end{gathered}
$$

where $F_{i}$ is the Jacobian of the dyanmics evaluated at the estimated mean value $\mu_{i-1}=\Sigma_{i-1}^{(-1)} \xi_{i-1}$.

Remark 1. In a multi robot scenario, where $\Sigma$ and $\xi$ characterize the probability distribution of all the MAVs, a distributed algorithm for the implementation of update equations (6)-(7) can be designed (see Section 3 and [1]). On the other hand, the prediction equations (8)-(9) are more complicated and they cannot be easily distributed. Nevertheless we will show that, once a delayed-state is considered, data obtained from proprioceptive sensors can be integrated using only the update equations (6)-(7).

Let us introduce the delayed-state

$$
X_{i}=\left(r_{0}, q_{0}, r_{1}, \ldots, r_{i}, q_{i}\right)
$$

containing all MAV poses until the $i$-th time step. The discretization of the dynamics equations over a $\Delta t$ time-step interval gives

$$
\begin{gathered}
r_{i+1}=r_{i}+v_{i} \Delta t \\
v_{i+1}=v_{i}+q_{i} \cdot \int_{i}^{i+\Delta t} A d t \cdot q_{i}^{*}+a_{g} \Delta t \\
q_{i+1}=q_{i}+\frac{1}{2} q_{i} \cdot \int_{i}^{i+\Delta t} \Omega d t
\end{gathered}
$$

From Equation (10) we can get 


$$
v_{i}=\left(r_{i+1}-r_{i}\right) / \Delta t
$$

and hence the following recursive formula holds

$$
r_{i+1}=2 r_{i}-r_{i-1}+\Delta t\left(q_{i} \cdot \int_{i}^{i+\Delta t} A d t \cdot q_{i}^{*}+a_{g} \Delta t\right),
$$

corresponding to a second order continuous-time evolution. Setting

$$
\tilde{A}_{i}=\int_{i}^{i+\Delta t} A d t
$$

and

$$
\tilde{\Omega}_{i}=\int_{i}^{i+\Delta t} \Omega d t
$$

the proprioceptive measurements can be regarded as delayed-state dependent functions:

$$
\begin{gathered}
\tilde{A}_{i}=h_{A}\left(r_{i-2}, r_{i-1}, r_{i}, q_{i}\right)=\frac{q_{i}^{*}\left(-a_{g} \Delta t^{2}+r_{i}-2 r_{i-1}+r_{i-2}\right) q_{i}}{\Delta t} \\
\tilde{\Omega}_{i}=h_{\Omega}\left(q_{i-1}, q_{i}\right)=2 q_{i-1}^{*}\left(q_{i}-q_{i-1}\right) .
\end{gathered}
$$

In other words, $\tilde{A}_{i}$ and $\tilde{\Omega}_{i}$ are functions of the state $X_{i}$ to be estimated; moreover, since we are considering the discrete dynamics given by (12)-(13), there is no need to include the MAV speed $v$ into the state vector $X_{i}$.

Due to these considerations, we are allowed to integrate proprioceptive data using (6)-(7) instead of (8)-(9), with a consequent reduction of computational cost in the estimation algorithm.

For nonlinear measurements equation (7) involves the mean value and hence information matrix inversion is required; nevertheless in many situation, due to the sparsity of such matrix, a partial state recovery is sufficient in order to guarantee a good estimate (see [2]). Whole state recovering can be obtained using for example the Conjugate Gradients algorithm (see [18]) or the Givens rotations factorization (see [8]). We point out that at any update step, i.e. when a true exteroceptive measurement is performed, the size of the delayed-state vector $X$ increases by $3+4=7$.

\subsection{Projection filter: integration of ideal constraints}

As mentioned in the introduction, the quaternion structure is redundant for the problem we are considering and this may lead to a loss of information. To avoid this problem we have assumed that the quaternion $q$ is unitary. On the other hand, if the discrete dynamics (12) is considered, such property is no longer preserved. Anyway, we can take into account the norm invariance of $q_{i}$ imposing an ideal constraint with a fake observation given by the function 


$$
h_{0}(q)=1-q_{t}^{2}+q_{x}^{2}+q_{y}^{2}+q_{z}^{2}
$$

in other words, we can regard the norm constraint as the measurement

$$
z_{i}=h_{0}\left(q_{i}\right)=0
$$

Integration of such fake measurement can be performed with the projection filter (see [14]).

\section{The Cooperative Case}

\subsection{The System}

We consider now a fleet of $N>1$ MAVs, each one having the characteristics described in Section 2. Let us denote by $\left(r^{(k)}, q^{(k)}\right)$ the coordinates of the $k$-th MAV; the discrete dynamics is given by

$$
\begin{gathered}
r_{i+1}^{(k)}=2 r_{i}^{(k)}-r_{i-1}^{(k)}+\Delta t\left(q_{i}^{(k)} \cdot \int_{i}^{i+\Delta t} A^{(k)} d t \cdot\left(q_{i}^{(k)}\right)^{*}+a_{g} \Delta t\right) \\
q_{i+1}^{(k)}=q_{i}^{(k)}+\frac{1}{2} q_{i}^{(k)} \cdot \int_{i}^{i+\Delta t} \Omega^{(k)} d t .
\end{gathered}
$$

Each MAV, in addition to the measurement model (4), may perform relative observation; the general multi robot observation equation can be written as

$$
z_{i}^{(k)}=h^{(k)}\left(r_{i}^{(1)}, q_{i}^{(1)}, \ldots, r_{i}^{(k)}, q_{i}^{(k)}, \ldots, r_{i}^{(N)}, q_{i}^{(N)}\right)
$$

Simple and common examples of relative observations are distance measures. If the $k$-th MAV measures its own distance from the $j$-th MAV, the observation is given by

$$
z_{i}^{(k)}=\left(r_{i, x}^{(k)}-r_{i, x}^{(j)}\right)^{2}+\left(r_{i, y}^{(k)}-r_{i, y}^{(j)}\right)^{2}+\left(r_{i, z}^{(k)}-r_{i, z}^{(j)}\right)^{2} .
$$

\subsection{The Distributed EIF}

In [1] it is shown that delayed-states allow to distribute the estimation process over the entire MAVs network. In particular the authors explain how to recover the global belief from the local belief of each network node and remark that the same operation with standard (non delayed) states is not possible at all. We will follow a similar approach, with a slightly different communication and data fusion algorithm.

When the exploration starts, each MAV begins to integrate the information provided 
by its own sensors by equation (6)-(7) as described before. In particular for any measurement, the incoming data are stored in the bottom-right block of the information matrix and, as a consequence, in the last entries of the information vector:

$$
\begin{gathered}
\Sigma_{i-1} \rightarrow \Sigma_{i}=\left(\begin{array}{cc}
\Sigma_{i-1} & 0^{7(i-1) \times 7} \\
0^{7 \times 7(i-1)} & 0^{7 \times 7}
\end{array}\right)+\left(\begin{array}{cc}
0^{7(i-3) \times 7(i-3)} & 0^{7(i-3) \times 21} \\
0^{21 \times 7(i-3)} & \Sigma_{o b s}
\end{array}\right) \\
\xi_{i-1} \rightarrow \xi_{i}=\left(\begin{array}{c}
\xi_{i-1} \\
0^{7 \times 1}
\end{array}\right)+\left(\begin{array}{c}
0^{7(i-3) \times 1} \\
\xi_{o b s}
\end{array}\right) .
\end{gathered}
$$

Suppose that after $i_{1}$ updating time-steps for the $j_{1}$-th MAV and $i_{2}$ steps for the $j_{2}$ th MAV a relative measurement occurs and for sake of simplicity suppose that $j_{1}<$ $j_{2}$. Each MAV has to increase the size of the information matrix and information vector in order to store the new data. The process is carried out following the steps described below:

1. State augmentation. The states of the two MAVs are increased in order to have the same size $7\left(i_{1}+i_{2}\right)$; this can be done adding a suitable number of zeros in the information matrix and information vector.

$$
\begin{aligned}
& \Sigma_{\left(j_{1}\right), i_{1}} \rightarrow\left(\begin{array}{cc}
\Sigma_{\left(j_{1}\right), i_{1}} & 0^{7 i_{1} \times 7 i_{2}} \\
0^{7 i_{2} \times 7 i_{1}} & 0^{7 i_{2} \times 7 i_{2}}
\end{array}\right), \quad \xi_{\left(j_{1}\right), i_{1}} \rightarrow\left(\begin{array}{c}
\xi_{\left(j_{1}\right), i_{1}} \\
0^{7 i_{2} \times 1}
\end{array}\right) \\
& \Sigma_{\left(j_{2}\right), i_{2}} \rightarrow\left(\begin{array}{cc}
0^{7 i_{1} \times 7 i_{1}} & 0^{7 i_{1} \times 7 i_{2}} \\
0^{7 i_{2} \times 7 i_{1}} & \Sigma_{\left(j_{2}\right), i_{2}}
\end{array}\right), \quad \xi_{\left(j_{2}\right), i_{2}} \rightarrow\left(\begin{array}{c}
0^{7 i_{1} \times 1} \\
\xi_{\left(j_{2}\right), i_{2}}
\end{array}\right)
\end{aligned}
$$

2. Relative estimation. The information from relative observations are integrated using the standard update equations (6)-(7). Correlation between the estimates on the last poses of the MAVs may appear, so that the updated matrices may be not block-diagonal.

$$
\Sigma_{\left(j_{1}\right), i_{1}} \rightarrow\left(\begin{array}{cc}
\Sigma_{\left(j_{1}\right), i_{1}} & * \\
* & *
\end{array}\right), \quad \xi_{\left(j_{1}\right), i_{1}} \rightarrow\left(\begin{array}{c}
\xi_{\left(j_{1}\right), i_{1}} \\
*
\end{array}\right)
$$




$$
\Sigma_{\left(j_{2}\right), i_{2}} \rightarrow\left(\begin{array}{cc}
* & * \\
* & \Sigma_{\left(j_{2}\right), i_{2}}
\end{array}\right), \quad \xi_{\left(j_{2}\right), i_{2}} \rightarrow\left(\begin{array}{c}
* \\
\xi_{\left(j_{2}\right), i_{2}}
\end{array}\right)
$$

3. Data fusion. A communication is established between the MAVs and they exchange their stored data. The data fusion scheme is a non negligible theoretical issue: as a matter of fact, if the process is carried out taking simply the sum of the contributions from each MAV, estimation errors may arise due to adding several times the same information. Following [1], we have adopted a fusion algorithm based on a convex combination of the data:

$$
\begin{aligned}
& \Sigma_{\left(j_{1}\right), i_{1}} \rightarrow \omega \Sigma_{\left(j_{1}\right), i_{1}}+(1-\omega) \Sigma_{\left(j_{2}\right), i_{2}}, \quad \xi_{\left(j_{1}\right), i_{1}} \rightarrow \omega \xi_{\left(j_{1}\right), i_{1}}+(1-\omega) \xi_{\left(j_{2}\right), i_{2}} \\
& \Sigma_{\left(j_{2}\right), i_{2}} \rightarrow(1-\omega) \Sigma_{\left(j_{1}\right), i_{1}}+\omega \Sigma_{\left(j_{2}\right), i_{2}},
\end{aligned}
$$

As proved in [7], for any $0<\omega<1$, the above convex combinations lead to unbiased and consistent estimates, i.e. no overconfident estimate is performed and there is no overlapping of information.

\section{Performance Evaluation}

In order to validate our approach we perform simulations.

\subsection{The Simulated Environment}

The trajectories of the MAVs are generated randomly and independently one from each other. In particular, for every MAV, the motion is generated by generating randomly the linear and angular acceleration at $100 \mathrm{~Hz}$. Specifically, at each time step, the three components of the linear and the angular acceleration are generated as Gaussian independent variables with mean values $\mu_{a}$ and $\mu_{\dot{\Omega}}$ and with covariance matrices $P_{a}$ and $P_{\dot{\Omega}}$. By performing many simulations we remarked that the precision of the proposed strategy is almost independent of all these parameters. The simulations provided in this section are obtained with the following settings: $\mu_{a}=\mu_{\dot{\Omega}}=$ $[000]^{T}, P_{a}=\left[\begin{array}{ccc}\left(5 m s^{-2}\right)^{2} & 0 & 0 \\ 0 & 0 & 0 \\ 0 & 0 & 0\end{array}\right]$ and $P_{\dot{\Omega}}=\left[\begin{array}{ccc}\left(10 \mathrm{deg} \mathrm{s} s^{-2}\right)^{2} & 0 & 0 \\ 0 & \left.(10 \mathrm{deg} \mathrm{s})^{-2}\right)^{2} & 0 \\ 0 & 0 & \left(10 \mathrm{deg} \mathrm{s} s^{-2}\right)^{2}\end{array}\right]$ 
We adopt many different values for the initial MAV positions orientations and speeds. We also consider different scenarios corresponding to a different number of MAVs.

Starting from the accomplished trajectories, the true angular speed and the linear acceleration are computed at each time step of $0.01 s$ (respectively, at the time step $i$, we denote them with $\Omega_{i}^{\text {true }}$ and $A_{i}^{\text {true }}$ ). Starting from them, the IMU sensors are simulated by generating randomly the angular speed and the linear acceleration at each step according to the following: $\Omega_{i}=N\left(\Omega_{i}^{\text {true }}, P_{\Omega_{i}}\right)$ and $A_{i}=N\left(A_{i}^{\text {true }}-A_{g}, P_{A_{i}}\right)$ where $N$ indicates the Normal distribution whose first entry is the mean value and the second one its covariance matrix and $P_{\Omega_{i}}$ and $P_{A_{i}}$ are the covariance matrices characterizing the accuracy of the IMU; finally, $A_{g}$ is the gravity acceleration expressed in the local frame. In all the simulations we set both $P_{A_{i}}$ and $P_{\Omega_{i}}$ diagonal matrices. In the results here provided they are set as follows: $P_{A_{i}}=\left[\begin{array}{ccc}\left(0.1 \mathrm{~ms}^{-2}\right)^{2} & 0 & 0 \\ 0 & \left(0.1 \mathrm{~ms}^{-2}\right)^{2} & 0 \\ 0 & 0 & \left(0.1 \mathrm{~ms}^{-2}\right)^{2}\end{array}\right]$ and $P_{\Omega_{i}}=$ $\left[\begin{array}{ccc}\left(10 \operatorname{deg~} s^{-1}\right)^{2} & 0 & 0 \\ 0 & \left(10 \operatorname{deg~s} s^{-1}\right)^{2} & 0 \\ 0 & 0 & \left(10 \operatorname{deg~s} s^{-1}\right)^{2}\end{array}\right]$ for every step $i$.

The MAVs are also equipped with GPS and range sensors. The GPS provides the position of the MAV with a Gaussian error whose covariance is a diagonal matrix and whose components are equal to $25 \mathrm{~m}^{2}$. The GPS data are delivered at $5 \mathrm{~Hz}$. Finally, the range sensors provide the distances among the MAVs at $2 \mathrm{~Hz}$ and the measurement errors are normally distributed with variance $(0.01 \mathrm{~m})^{2}$.

\subsection{Results}

We provide some of the results obtained with the previous settings and by simulating $N$ MAVs. In particular, we consider the case of $N=3$ and $N=5$. Furthermore, we consider separately the cases when the estimation is performed by only integrating the IMU data, by combining the IMU data with the GPS data and by combining all the sensor data. Finally, in order to evaluate the benefit of using the projection filter discussed in Section 2.4, we consider separately the cases when this filter is adopted and when it is not adopted.

Fig. 1a-b show the results obtained with three MAVs. The blue dots represent the ground truth. In fig. 1-a the magenta dots represent the GPS data and the black circles the trajectories estimated by only integrating the IMU data. It is clear that both IMU and GPS are very noisy and cannot be used separately to estimate the MAV trajectories. In fig. 1-b the green dots represent the trajectories estimated by fusing the IMU data and the GPS data with our proposed approach (EIF and projection filter). Finally, the red dots represent the result obtained by also fusing the range measurements. We remarked that the use of the range measurements further reduce the error. In particular, for the simulation in fig. 1a-b the position error averaged 
on all the three MAV and on all the time steps is equal to $0.6 m$ without the range measurements and $0.45 \mathrm{~m}$ with them. As expected, this improvement is still larger by increasing the number of MAVs (see for instance [17]). In fig. 1c-d the results obtained by using $5 \mathrm{MAVs}$ is shown. The position error obtained by also fusing the range measurements reduces to $0.2 \mathrm{~m}$.

Fig. 2 shows the benefit of using the Projection filter discussed in Section 2.4. In particular, in fig. 2 a the red circles represent the trajectories estimated by fusing all the sensor data and by running the Projection Filter at $5 \mathrm{~Hz}$ while in fig. $2 \mathrm{~b}$ the red circles represent the trajectories estimated without the use of the Projection Filter. As in the previous figures, the ground truth is represented with blue dots and the black dots represent the trajectories obtained by a simple integration of the IMU data.

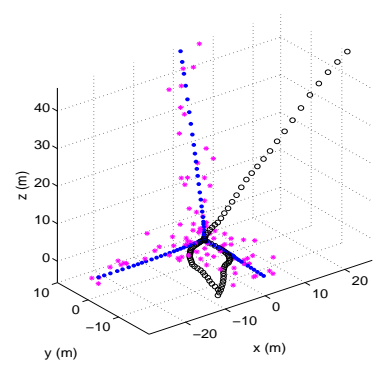

(a)

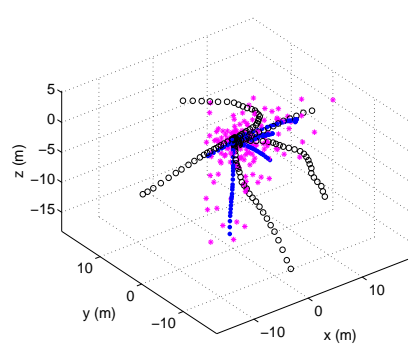

(c)



(b)

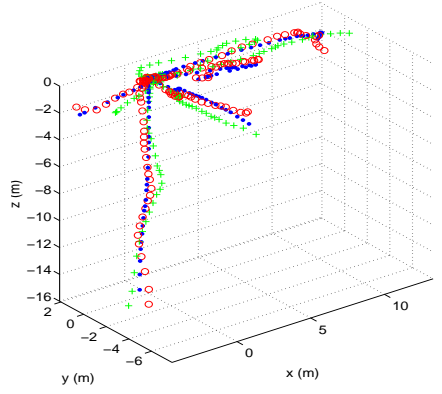

(d)

Fig. 1- Blue points represent true MAVs trajectories, black circles are the trajectories with only odometric estimates, magenta stars are the GPS data, green stars are the trajectory estimates without taking into account relative observations and red circles are the estimates with the complete distributed EIF. Figures (1a)-(1b) are the simulation of 3-MAV scenario, while in Figures (1c)-(1d) is plotted the evolution of a 5-MAV system. 


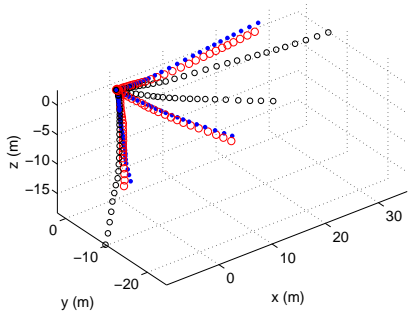

(a)

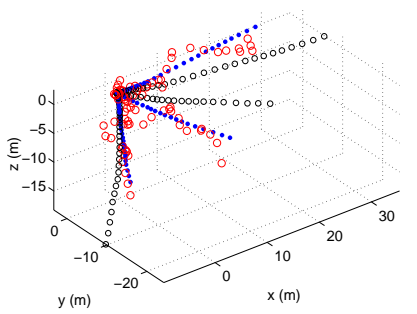

(b)

Fig. 2 - Blue points represent true MAVs trajectories, black circles are the estimated trajectories via odometry and red circles are the estimated trajectories with the EIF. Figure (2a) represents the simulation of a 3-MAV system; Figure ( $2 \mathrm{~b})$ represents the same scenario without taking into account the information provided by the projection filter.

\section{Conclusions}

In this paper we have discussed an approach to perform cooperative localization of a team of micro aerial vehicles equipped with inertial sensors (one accelerometer and one gyroscope) and exteroceptive sensors (GPS and range sensors). The approach is based on an Extended Information Filter whose implementation is distributed over the team members.

Two original contributions have been introduced. The former consists of a simple trick which allowed us to avoid the equations which characterize the prediction phase of the extended information filter. In particular, the information contained in the data provided by the inertial sensors is exploited by using the equations which characterize the perception step of the EIF. This allowed us to easily distributing the entire estimation process over all the team members. The latter contribution is the use of a projection filter which allowed exploiting the information contained in the geometrical constraints which arise as soon as the MAV orientations are characterized by unitary quaternions.

The performance of the proposed approach was evaluated by using synthetic data.

Acknowledgment: The research leading to these results has received funding from the European Community's Seventh Framework Programme (FP7/2007-2013) under grant agreement n. 231855 (sFly). 


\section{References}

1. J. Capitan, L. Merino, F. Caballero and Anbal Ollero, Delayed-state Information Filter for Cooperative Decentralized Tracking, ICRA, 2009, Kobe, Japan, pages 3865-3870.

2. R.M. Eustice, H. Singh and J.J. Leonard, Exactly Sparse Delayed-State Filters for ViewBased SLAM, IEEE Trans. on Robotics, vol. 22, n. 6, 2006, pages 1100-1114.

3. D. Fox, W. Burgard, H. Kruppa, S. Thrun, 2000, A Probabilistic Approach to Collaborative Multi-Robot Localization, Autonomous Robots 8, 2000, pages 325-344.

4. R. Grabowski, L.E. Navarro-Serment, C.J.J. Paredis, P.K. Khosla, 2000, Heterogeneous Teams of Modular Robots for Mapping and Exploration, Autonomous Robots, Vol. 8, n. 3, June 200?, pages 325-344.

5. A. Howard, M.J. Mataric and G.S. Sukhatme, "Localization for Mobile Robot Teams Using Maximum Likelihood Estimation", International Conference on Inteligent Robot and Systems (IROS02), Volume: 3 , 30 Sept.-5 Oct. 2002,Lausanne, pages 2849-2854.

6. E. Jones, A. Vedaldi and S. Soatto, Inertial Structure from Motion with Autocalibration, ICCU Workshop, 2007.

7. S.J. Julier and J.K. Uhlmann, A Non-divergent Estimation Algorithm in the Presence of Unknown Correlations, American Control Conference, 1997, Albuquerque, New Mexico, pages 2369-2373.

8. M. Kaess, A. Ranganathan and F. Dellaert, iSAM: Incremental Smoothing and Mapping, IEEE Trans. on Robotics, vol. 24, n.6, 2008, pages 1365-1378.

9. K. Kato, H. Ishiguro, M. Barth, "Identifying and Localizing Robots in a Multi-Robot System Environment" International Conference on Inteligent Robot and Systems (IROS99) 1999

10. R. Kurazume, R. Nagata and S. Hirose, Cooperative Positioning with Multiple Robots, IEEE International Conference on Robotics and Automation, 1994, pp. 1250-1257.

11. K.Y. Leung, T.D. Barfoot and H.H. Liu, Decentralized Localization of SparselyCommunicating Robot Networks: A Centralized-Equivalent Approach, IEEE Trans. on Robotics, vol. 26, n.1, 2010, pages 62-77.

12. A. Martinelli, F. Pont and R. Siegwart , "Multi-Robot Localization Using Relative Observations" International Conference on Robotics and Automation, April 2005, Barcellona, Spain.

13. A.I. Mourikis, S.I. Roumeliotis, "Optimal Sensing Strategies for Mobile Robot Formations: Resource-Constrained Localization", Robotics: Science and Systems June 8-11, 2005 Massachusetts Institute of Technology Cambridge, Massachusetts, USA

14. P. Newman, On the structures and solution of simultaneous localization and mapping problem, PhD thesis, Australian Center for Field Robotics, Sidney, 1999.

15. I.M. Rekleitis, G. Dudek and E.E. Milios, "Multi-robot cooperative localization: a study of trade-offs between efficiency and accuracy "International Conference on Inteligent Robot and Systems (IROSO2) Lausanne, Switzerland

16. S.I. Roumeliotis and G.A. Bekey, 2002, Distributed Multirobot Localization, IEEE Transaction On Robotics And Automation Vol 18, No.5, October 2002

17. S.I. Roumeliotis and I.M. Rekleitis, Propagation of Uncertainty in Cooperative Multirobot Localization: Analysis and Experimental Results, Autonomous Robots, vol. 17, n.1, 2004,pp. 41-54

18. J. Schewchuk, An Introduction to Conjugate Gradient Method without Agonizing Pain, Carnegie-Mellon University, Pittsburgh, PA, Tech. Report CMU-CS-94-125, 1994.

19. J.R. Spletzer and C.J. Taylor, "A Bounded Uncertainty Approach to Multi-Robot Localization" International Conference on Inteligent Robot and Systems (IROSO3) Las Vegas, USA, 2003

20. S. Thrun, W. Burgard and D. Fox, Probabilistic Robotics, MIT Press, Cambridge, MA, 2005. 